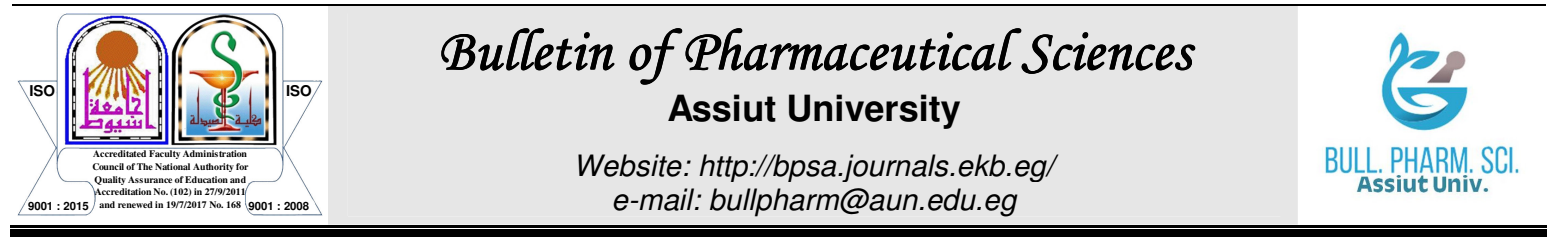

\title{
INSIGHTS INTO THE IMPACT OF FXR ACTIVATION ON HEPATIC AUTOPHAGY IN A NON-ALCOHOLIC STEATOHEPATITIS MODEL
}

\author{
Rasha A. Tawfiq ${ }^{1}$, Yasmeen M. Attia ${ }^{1}$, Noha N. Nassar ${ }^{2}$, Olfat A. Hammam², \\ Mohamed M. Elmazar ${ }^{1}$ and Dalaal M. Abdallah ${ }^{2 *}$ \\ ${ }^{1}$ Department of Pharmacology, Faculty of Pharmacy, The British University in Egypt, Cairo, \\ Egypt \\ ${ }^{2}$ Department of Pharmacology, Faculty of Pharmacy, Cairo University, Cairo, Egypt \\ ${ }^{3}$ Department of Pathology, Theodor Bilharz Research Institute, Cairo, Egypt
}

\begin{abstract}
Background: Multiple lines of evidence pointed to the role of dysbiosis, small intestinal bacterial overgrowth and altered intestinal permeability in promoting pro-inflammatory events in the liver leading to the progression of steatosis to non-alcoholic steatohepatitis (NASH). The pivotal involvement of farnesoid-X-receptor (FXR) in maintaining intestinal homeostasis and combating hepatic inflammation was previously established. Nonetheless, the role of hepatic autophagy in NASH pathogenesis and treatment remains controversial. The present study aimed at investigating whether the effect of the FXR agonist, obeticholic acid, on ameliorating NASHrelated incidents is related to an impact on hepatic autophagy. Methods: Swiss albino mice were fed an atherogenic high fat diet (Ath-HFD) with dextran sulfate sodium (DSS) in drinking water to induce NASH. Obeticholic acid (5 mg/kg/day, p.o.) was given for 28 days, starting at day 64 post NASH initiation. Histopathological examination of liver and colon samples was performed. Inflammatory markers, $I L-1 \beta, I L-6, I F N-\gamma$ and $T N F-\alpha$, besides adiponectin, were assessed in the liver. Autophagy genes, ULK1, BECN-1 and ATG5, were assessed by RT-PCR in hepatic tissues. Results: Histopathological changes observed in the liver and colon of the positive control group (Ath-HFD/DSS) were significantly ameliorated after treatment. No noticeable changes were reported in adiponectin and inflammatory markers following treatment except for a partial enhancement in IFN- $\gamma$. Though ULK1 and BECN-1 gene expression tended to increase after treatment with obeticholic acid compared to Ath-HFD/DSS group, ATG5 mRNA was almost restored. Conclusion: Obeticholic acid ameliorated NASH partially through autophagy and IFN- $\gamma$ enhancements in the liver.
\end{abstract}

\section{INTRODUCTION}

Nonalcoholic fatty liver disease (NAFLD) is a condition in which fats accumulate in the liver of patients with no history of alcohol abuse $^{1}$. It is classified into simple steatosis and nonalcoholic steatohepatitis (NASH). The progressive fibrotic liver disease, NASH, may advance to cirrhosis and its complications, hepatocellular carcinoma and end-stage liver disease $^{2}$. Indeed, NASH is considered a risk factor for liver- and non-liver-related morbidity and mortality ${ }^{3}$. The classical NASH pathogenesis theory stated that NASH develops in two steps but more recently, a multiple hit theory was launched ${ }^{4}$. At first, the healthy liver becomes steatotic as a consequence of insulin resistance that sequentially causes an increase in the transport of fatty acids from the adipose tissue $^{5}$. Visceral adipose tissue participates in triggering hepatic injury through alteration of fats and glucose metabolism, causing fat accumulation in the liver and therefore creating a pro-inflammatory ambiance ${ }^{6}$. Bacterial lipopolysaccharides, as an insult, lead to cytokines production, in particular tumor necrosis factor- $\alpha$ (TNF- $\alpha$ ), thus potentiating oxidative stress to exacerbate liver damage ${ }^{7}$.

Received in 29/10/2020 \& Accepted in 14/11/2020 
Evidence exists that both intestinal epithelial barrier dysfunction and microbial dysbiosis contribute to NASH pathogenesis ${ }^{8}$. Since $70 \%$ of the blood reaching the liver comes from the portal vein, delivering the blood coming from the gut to the liver, the latter becomes vulnerable to bacterial antigens and toxins. ${ }^{5}$. Human intestinal microbiome has indispensable roles in vitamin biosynthesis besides degradation of bile acids and carbohydrates as well as maintaining intestinal integrity ${ }^{9}$ In physiological conditions, incremental amounts of translocated bacterial components reach the liver which can be therefore endured by the hepatic immune system, a phenomenon known as liver tolerance $^{10-12}$. Intestinal barrier disruption, on the other hand, provokes the development of a leaky gut, which contributes to bacterial translocation, viz. the emigration of bacteria or their components from the gut lumen to the mesenteric lymph nodes and other sites 5 Additionally, increased bacterial translocation through the gut-liver axis may cause a distortion in hepatic homeostasis enhancing inflammatory incidents via activating the innate immune response ${ }^{13}$. Simultaneously, small intestinal bacterial overgrowth occurs in 20$75 \%$ of patients suffering from chronic liver disease ${ }^{5}$. Therefore, altered intestinal permeability, bacterial overgrowth and immunity disorders are all contributing factors in the pathogenesis and progression of $\mathrm{NASH}^{14}$. It is noteworthy that bacterial translocation is not restricted to late stages but also has a role in disease progression from a very early stage. Hence, attempts to restore gut microbiota and intestinal integrity have been sought as possible interventions for the treatment of liver diseases ${ }^{15}$.

Bile acids (BAs) are steroid acids that are synthesized in the liver and stored in the gall bladder to be released into the intestine to aid fat digestion ${ }^{16}$. Furthermore, BAs affect the microflora through nuclear receptors like farnesoid-X-receptor (FXR) as BAs are considered the physiological ligands of FXR, which is responsible for glucose and lipid homeostasis ${ }^{17}$. FXR is a nuclear hormone receptor expressed in tissues responsible for bilirubin metabolism, like liver, intestine and kidneys. FXR has a pivotal role in carbohydrates and lipid metabolism, improvement of hyperlipidemia in addition to its role in hepatic regeneration after injury ${ }^{18}$. FXR activation was found to impede fatty liver injury in different NASH models ${ }^{19 \& 20}$. Obeticholic acid is a 6 $\alpha$-ethyl derivative of the naturally occurring endogenous BA, chenodeoxycholic acid, and a selective FXR agonist that is almost 100 folds more potent than chenodeoxycholic acid, and thus is sought as a potential strategy in treating NASH patients $^{18}$. It is worth mentioning that obeticholic acid has been approved by the FDA as an orphan drug in May 2016 for the use in primary biliary cholangitis ${ }^{21}$. Intestinal microbiome affects BA composition along with the expression of genes downstream of $\mathrm{FXR}^{17}$. Collectively, these data show that BAs can alter gut microbiota and vice versa, which could play a role in treating NAFLD ${ }^{17 \& 22}$. Thus, restoring the intestinal barrier integrity and gut microbiota composition might be pursued as a therapeutic strategy in NASH patients ${ }^{8}$.

NASH pathogenesis is not fully understood $^{5}$, however, the role of an altered FXR expression in the progression of simple steatosis to NASH is well characterized ${ }^{23}$. On the other hand, the exact role of FXR activation on hepatic autophagy and how it reflects on NASH-related events triggered by gut leakage still needs to be construed. This study, therefore, aimed at investigating the potential influence of obeticholic acid as an FXR agonist on modulating autophagy in the liver in an experimental model of NASH induced by an atherogenic high fat diet (Ath-HFD) with dextran sulfate sodium (DSS) in drinking water.

\section{MATERIALS AND METHODS}

\section{Experimental Animals}

Laboratory in-bred Swiss albino male mice, 25-35 g weight, were used in the current study. Except for the periods of induction of colon inflammation, animals had access to drinking water ad libitum with standard chow (SC) diet or Ath-HFD. All animals were housed under controlled conditions of temperature $\left(23 \pm 2^{\circ} \mathrm{C}\right)$ and humidity $(60 \pm 10 \%)$ with $12 \mathrm{~h}$ dark/light cycles and were receive humane care in compliance with the Research Ethics Committee guidelines (Faculty of Pharmacy, Cairo University, Cairo, Egypt). 


\section{Experimental model}

A combined model of chronic colon inflammation and dietary NASH paradigm was used based on ${ }^{7}$ model. Colon inflammation was induced by administration of DSS (TdB Consultancy AB, Uppsala, Sweden) in drinking water alternating with normal drinking water throughout the whole experimental period, while NASH was induced by the Ath-HFD. Notably, DSS-mediated colon inflammation results in increased bacterial product concentrations in portal vein due to impaired colonic barrier function and consequently, hepatic inflammation might occur ${ }^{7}$. Moreover, the dietary Ath-HFD NASH model reflects both hepatic histopathology and pathophysiology of human $\mathrm{NASH}^{24}$.

Experiments were performed using a total of 30 mice which were randomly allocated into 3 experimental groups $(n=10)$; where animals of group I were fed SC ad libitum (negative control), group II mice were fed Ath-HFD ad libitum and DSS in drinking water throughout the experimental period in cycles (AthHFD/DSS); and group III were fed Ath-HFD and DSS in cycles as described in group II in addition to treatment with the FXR agonist, obeticholic acid $(5 \mathrm{mg} / \mathrm{kg} / \mathrm{day}$, p.o.) starting 28 days before experiment termination (AthHFD/DSS+OCA group). On the day of sacrifice, mice were deeply anesthetized, organs were harvested and a portion of the tissue were fixed in $10 \%$ formal-saline or snap frozen in liquid nitrogen and stored at $-80^{\circ} \mathrm{C}$.

\section{Methods}

\section{Histopathological examination}

Hepatic and colon paraffinized sections $(4 \mu \mathrm{m})$ were stained with hematoxylin \& eosin (H\&E). The histopathological investigation was executed using light microscope at X100 (for colon) and X200 (for liver) magnification powers in a blind fashion.

\section{Gene expression studies}

Using real time reverse transcription polymerase chain reaction (RT-PCR) technique on frozen liver tissue, the relative mRNA expression of Unc-51 like autophagy activating kinase (ULK1), beclin-1 (BECN-1) and Autophagy related 5(ATG5) genes were assessed. RNA extraction (Qiagen, Hilden, Germany) and cDNA synthesis (Qiagen,
Hilden, Germany) were performed followed by quantitative PCR using the SYBR green PCR kit (Qiagen, Hilden, Germany). The results were obtained as $2^{\wedge} \_\Delta \Delta \mathrm{Ct}$.

\section{Enzyme-linked immunosorbent assays (ELISA) \\ Adiponectin assay}

The fat-burning adipokine, adiponectin content, was determined in liver homogenates using sandwich enzyme-linked immunosorbent assay (ELISA) Mouse Adiponectin ELISA Kit PicoKine ${ }^{\mathrm{TM}}$ (Boster Biological Technology, CA, USA). Hundred $\mu 1$ of tissue homogenate, prepared as 1:4 in saline, diluted in sample buffer, was added to a 96 wells plate coated with the capture antibody and incubated for 1.5 $\mathrm{hr}$ at $37^{\circ} \mathrm{C}$ parallel to controls and standards. After discarding plate content, biotinylated detection antibody was added and incubated for $1 \mathrm{hr}$ at $37^{\circ} \mathrm{C}$. After discarding and washing the plate $3 \mathrm{X}$, avidin-biotin-peroxidase complex was added and incubated for $0.5 \mathrm{hr}$ at $37^{\circ} \mathrm{C}$. Finally, a coloring agent was added after discarding and washing the plate that was allowed for incubation $\left(15 \mathrm{~min}\right.$ at $\left.37^{\circ} \mathrm{C}\right)$, then the reaction was stopped by adding stop solution and the reaction was read at $450 \mathrm{~nm}$ using a microplate reader (BioTek ELx800 Microplate Reader, Vermont, USA).

\section{Inflammatory markers IL-1 $\beta$, IL-6, IFN- $\gamma$ and TNF- $\alpha$ ELISA assays}

The homogenate of liver tissue of each group was prepared (1:4 in saline) to be assessed for protein concentration of interleukin-1 $\beta \quad$ (IL-1 $\beta), \quad$ IL-6, interferongamma (IFN- $\gamma$ ), TNF- $\alpha$ using commercial ELISA which were performed according to the manufacturers' instructions of Mouse PicoKine $^{\mathrm{TM}}$ ELISA Kits (Boster Biological Technology, CA, USA) and as previously mentioned.

\section{Statistical analysis}

Results were analyzed using one-way analysis of variance (ANOVA) followed by Tukey's post hoc test at statistical significance $(p<0.05$ ) by GraphPad Prism Version 6 (GraphPad prism Software, CA, USA). Data are shown as mean + standard deviation of mean (SDM). 


\section{RESULTS AND DISCUSSION}

\section{Results}

Obeticholic acid ameliorates NASH in liver histopathologically

Liver sections in the Ath-HFD/DSS group showed marked hepatocellular ballooning with fat droplets and macrovesicular steatosis, which was almost resolved in the obeticholic acid-treated group (Fig. 1). The colon sections showed epithelial hyperplasia and inflammatory cells infiltration in the AthHFD/DSS group; however, mild colonic inflammation and almost normal liver architecture was observed following obeticholic acid administration (Fig. 1).

Hepatic adiponectin content following obeticholic acid treatment in Ath-HFD/DSS mice

Adiponectin in Ath-HFD/DSS mice was reduced significantly compared to negative control (Fig. 2). This reduction in hepatic adiponectin was not reversed after treatment with obeticholic acid.

\section{Hepatic inflammatory markers following obeticholic acid treatment in Ath-HFD/DSS mice \\ Upon investigating the protein levels of different inflammatory markers in liver tissue}

homogenates, a non-significant change was shown in IL- $1 \beta$, IL- 6 and TNF- $\alpha$ in the mice of Ath-HFD/DSS group compared to the negative control while IFN- $\gamma$ protein level was significantly decreased. On the other side, following obeticholic acid treatment, AthHFD/DSS-mediated IFN- $\gamma$ amelioration was in part hindered to reach a midway level between the negative and positive control values. Nonetheless, IL-1 $\beta$, IL- 6 and TNF- $\alpha$ showed non-significant changes following obeticholic acid in comparison with Ath-HFD/DSS group (Fig. 3).

Gene expression of ULK1, BECN-1 and ATG5 following obeticholic acid treatment in Ath-HFD/DSS mice

To almost similar extents, significant reductions in ULK1 and BECN-1 genes expression in the liver were observed in both Ath-HFD/DSS and obeticholic acid groups versus the negative control group (Fig. $4 \mathrm{~A} \&$ 4B). Regarding ATG5, a significant decrease in its gene expression was exhibited in the AthHFD/DSS group accompanied by a slight elevation following treatment which failed to achieve significance from the insult (Fig. 4C).
(A) Negative Control
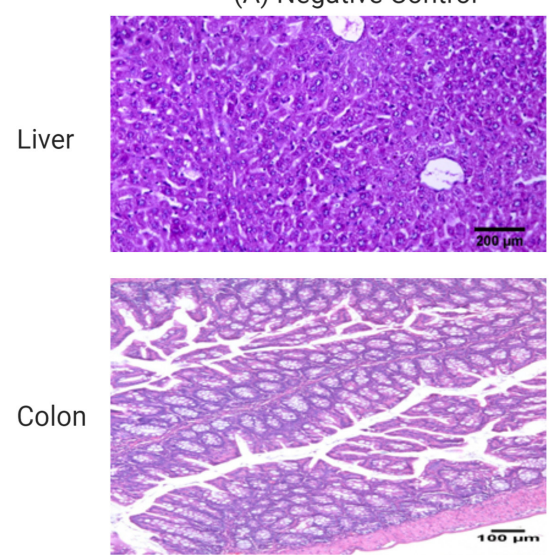

(B) Ath-HFD/DSS
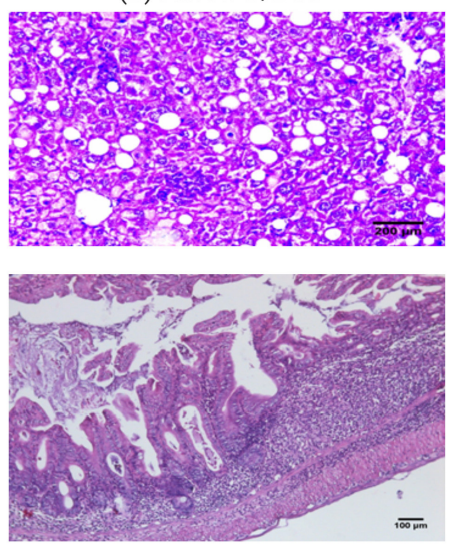

(C) Ath-HFD/DSS+OCA
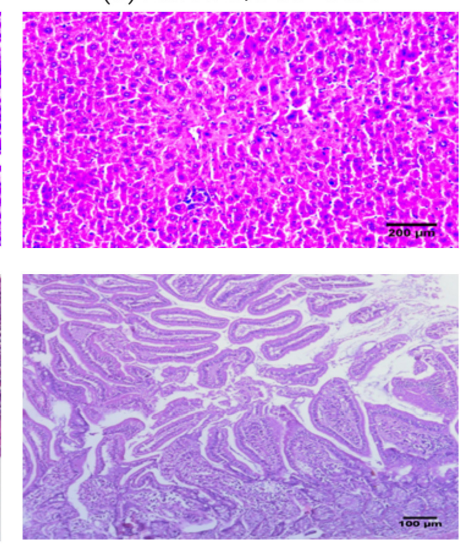

Fig. 1: Effect of 28 days obeticholic acid treatment on liver and colon histopathological alterations induced by Ath-HFD/DSS in mice.

Hematoxylin \& eosin (H\&E)-stained liver (x200) and colon (x100) sections from: (A) negative control, (B) AthHFD/DSS group and (C) Ath-HFD/DSS+OCA group. Ath-HFD: atherogenic high fat diet; DSS: dextran sulfate sodium; OCA: obeticholic acid. 


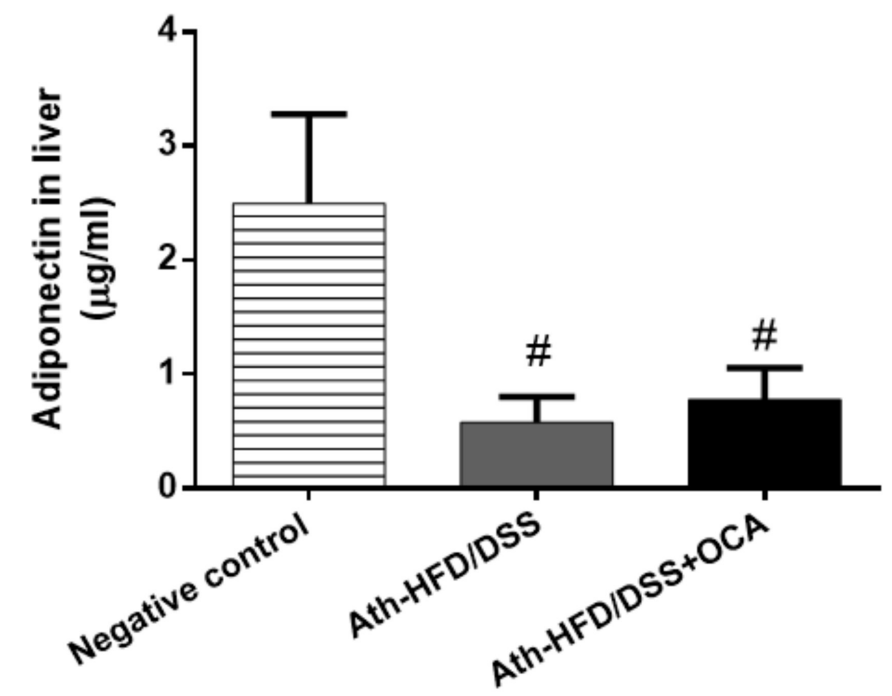

Fig. 2: Hepatic adiponectin content in Ath-HFD/DSS mice treated for 28 days with obeticholic acid.

Quantification was estimated using ELISA. Values are presented as means + SDM. Statistical analysis was performed using one-way ANOVA followed by Tukey's post hoc test. " Significant difference $v s$ negative control group at $p<0.05$. Ath-HFD: atherogenic high fat diet; DSS: dextran sulfate sodium; OCA: obeticholic acid.

(A)

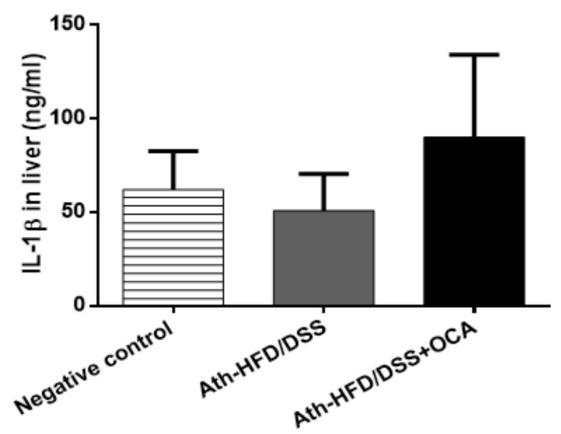

(C)

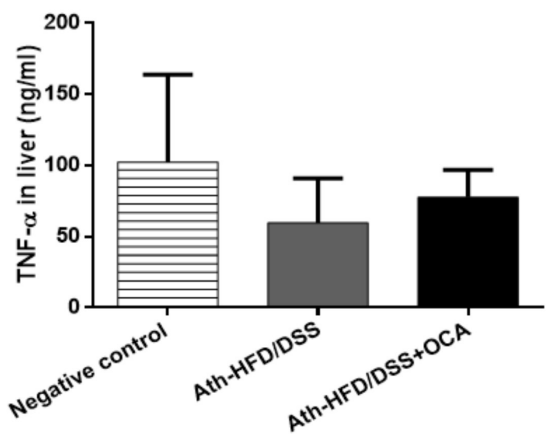

(B)

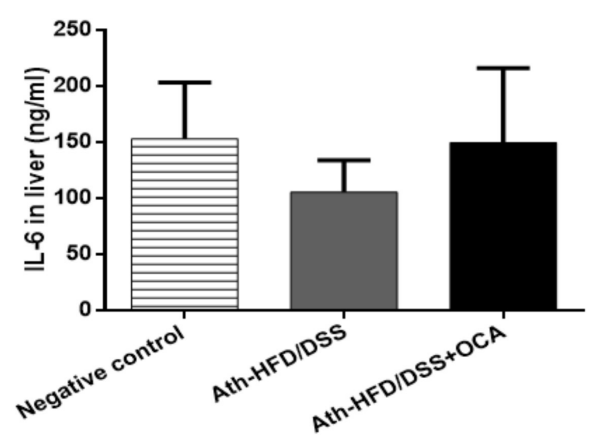

(D)

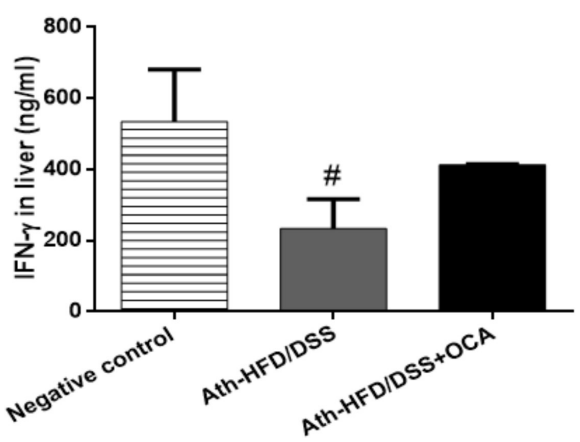

Fig. 3: Effect of 28 days obeticholic acid treatment on hepatic inflammatory markers in Ath-HFD/DSS mice.

Protein levels of A) IL-1 $\beta$, B) IL-6, C) TNF- $\alpha$ and D) IFN- $\gamma$ in liver tissue homogenates. Quantification was estimated using ELISA. Values are presented as means + SDM. Statistical analysis was performed using oneway ANOVA followed by Tukey's post hoc test. \# Significant difference vs negative control group at $p<0.05$. AthHFD: atherogenic high fat diet; DSS: dextran sulfate sodium; IFN- $\gamma$ : interferon-gamma; IL: interleukin, OCA: obeticholic acid; TNF- $\alpha$ : tumor necrosis factor-alfa. 
(A)

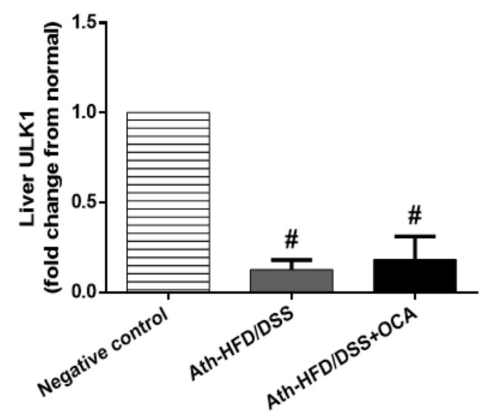

(B)

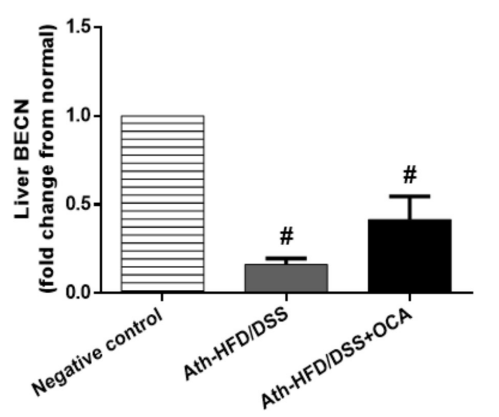

(C)

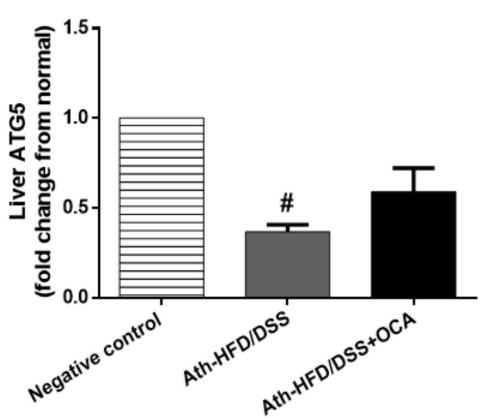

Fig. 4: Effect of 28 days obeticholic acid treatment on hepatic gene expressions of ULK1, BECN-1 and ATG5 in Ath-HFD/DSS mice.

Gene expression of (A) ULK1, (B) BECN-1 and (C) ATG5 was performed using RT-PCR. Values are presented as means + SDM. Values are inverse of square root transformation of means for (B \& C). Statistical analysis was performed using one-way ANOVA followed by Tukey's post hoc test. \#Significant difference vs negative control group at $p<0.05$. ATG5: Autophagy related 5; Ath-HFD: atherogenic high fat diet; DSS: dextran sulfate sodium; OCA: obeticholic acid; RT-PCR: reverse transcription polymerase chain reaction; ULK1: Unc-51 like autophagy activating kinase.

\section{Discussion}

The current study demonstrated obeticholic acid potential to ameliorate NASHinduced aberrant histological alterations through induction of autophagy and augmentation of INF- $\gamma$ anti-inflammatory effect. Whilst the drug neither modulated AthHFD/DSS effects on adiponectin nor proinflammatory cytokines (TNF- $\alpha$, IL- $1 \beta$ and IL-6) in the injured liver, it was capable of partially enhancing IFN- $\gamma$. This finding coincided with a tendency towards increase in ULK1 and BECN-1 gene expression in the treated group, whereas ATG5 mRNA was almost restored indicating its positive outcome on autophagy.

In the present study, an experimental model of Ath-HFD combined with DSS was used to induce hepatic injury accompanied by gut leakage. This was manifested in the observed inflammatory cells infiltration and epithelial hyperplasia in colon accompanied by hepatocellular ballooning in liver, as demonstrated by histopathological evaluation. On the other hand, obeticholic acid-treated group showed normal architecture of liver sections in mice and mild inflammatory cells infiltration in colon indicating the aptitude of the FXR agonist in ameliorating NASH induced by gut leakage, besides the associated colitis. These findings are in line with previous relevant reports ${ }^{25}$.
Adiponectin level in liver tissue is considered a negative biomarker for $\mathrm{NASH}^{26}$. Adiponectin is an adipokine that acts as a fatburning hormone and accordingly it showed a significant decrease in Ath-HFD/DSS group to comply with the histopathological data that confirmed the hallmarks of NASH, as previously mentioned. Following obeticholic acid treatment, this decrease was barely ameliorated as it showed slight insignificant increase $(\sim 35 \%)$ in adiponectin level in spite of the currently documented improvement in liver histopathology. This slight elevation might be attributed to the nature of adiponectin as it is not a sole marker in diagnosing NASH patients due to the vast variation in its results ${ }^{26}$. Indeed, the present results may confirm this perspective since the alteration on the histological level after treatment was not reflected on adiponectin levels.

In the present study, we were interested in investigating the effect of obeticholic acid on the inflammatory cytokines in the liver. In this context, a non-significant effect of obeticholic acid on the inflammatory cytokines, IL- $1 \beta$, IL-6 and TNF- $\alpha$, versus the Ath-HFD/DSS group was observed. Notably, the Ath-HFD/DSS group showed also no alterations in these cytokines compared to the negative control group. While previous studies revealed elevation in cytokines concentrations in the plasma of NASH patients ${ }^{23}$, this was not the 
case herein. Such a discrepancy could be explained in view of the model used, where in the current study DSS was added as an insult to aid in gut leakage to enhance the dietary NASH induced by high fat diet. Therefore, the repetitive cycles and long periods of DSS administration may have led to the potentiation of an adaptive mechanism or desensitization of the immune system towards an exaggerated inflammatory response induced towards the bacterial products translocated through the gutliver axis as previously described ${ }^{7}$. These authors reported no noticeable changes in the inflammatory cytokines in the mesenteric lymph nodes in a murine NASH model with colitis, supporting the present data. It was also believed that the effect on the inflammatory cytokines following DSS-induced inflammation is strain-specific ${ }^{25}$, where in Balb/C mice a rapid healing of inflammation has been observed following DSS withdrawal, an effect that might fit here.

The role of IFN- $\gamma$ in NASH pathogenesis is complex and controversial. Although IFN- $\gamma$ is ascribed as a very potent proinflammatory cytokine $^{27}$, other studies demonstrated its critical role in modulating innate and adaptive immune responses and its protective character against a plethora of insults ${ }^{28 \& 29}$ including $\mathrm{NASH}^{30}$. Indeed, IFN- $\gamma$ has been reported to accumulate neutrophils and macrophages in the liver $^{27}$ augment progression of liver injury into fibrosis $^{31}$ and was shown to increase in a model of methionine and choline-deficient high fat $\operatorname{diet}^{32}$ to highlight its injurious aptitude. Nonetheless, in line with its protective role, the decrease observed in IFN- $\gamma$ levels in this model was previously reported ${ }^{30}$. This study elucidated a paradigm shift towards IFN- $\gamma$ in the immunopathogenesis of NAFLD in obese IL-10/IL-4-deficient mice fed a HFD for 40 weeks confirming its protective potential in this setting. Moreover, increased hepatic expression of IFN- $\gamma$ was observed in wild mice, whereas IFN- $\gamma$-deficient mice fed a HFD showed accelerated NASH development with fibrosis in the aforementioned study to further support its benefit. This conclusion, therefore, acknowledges the enhancement of IFN- $\gamma$ by obeticholic acid as a factor of paramount importance in preserving/restoring liver architecture, as reported in the present study.
Though its association with NAFLD is still disputable, autophagy is considered as a target of interest in NAFLD drug development $^{33}$. Thus, on assessing the expression of autophagy genes in the livers of Ath-HFD/DSS, an inhibition of autophagy was noticed. This is consistent with other reports discussing the inhibition of the autophagic flux in $\mathrm{NASH}^{34 \& 35}$. In the current work, after treatment with obeticholic acid, ULK1 and BECN-1 gene expression showed a subtle increase that failed to reach significant levels whereas that of ATG5 was in part enhanced compared to the Ath-HFD/DSS-mediated suppression. The current results are in line with previous studies reporting the induction of autophagy in the liver following FXR activation $^{36}$ but in contrary to others showing its inhibition under similar experimental conditions $^{37 \& 38}$.

Overall, this study concludes that obeticholic acid impact on NASH induced by gut leakage depends partially on two strategies; IFN- $\gamma$ enhancement and autophagy induction in the liver.

\section{REFERENCES}

1- A. N. Mavrogiannaki and I. N. Migdalis, "Nonalcoholic fatty liver disease, diabetes mellitus and cardiovascular disease: Newer data", International Journal of Endocrinology, 2013, 1-8 (2013).

2- R. J. Wong, M. Aguilar, R. Cheung, R. B. Perumpail, S. A. Harrison, Z. M. Younossi and A. Ahmed, "Nonalcoholic steatohepatitis is the second leading etiology of liver disease among adults awaiting liver transplantation in the United States", Gastroenterology, 148, 547-555 (2015).

3- L. Brodosi, F. Marchignoli, M.L. Petroni and G. Marchesini, "NASH: A glance at the landscape of pharmacological treatment", Annals of Hepatology, 15, 673-681 (2016).

4- E. Buzzetti, M. Pinzani and E. A. Tsochatzis, "The multiple-hit pathogenesis of non-alcoholic fatty liver disease (NAFLD)", Metabolism: Clinical and Experimental, 65, 1038-1048 (2016). 
5- D. Compare, P. Coccoli, A. Rocco, O. M. Nardone, S. de Maria, M. Cartenì and G. Nardone, "Gut-liver axis: The impact of gut microbiota on non alcoholic fatty liver disease", Nutrition, Metabolism and Cardiovascular Diseases, 22, 471-476 (2012).

6- L. E. Armstrong and G. L. Guo, "Role of FXR in liver inflammation during nonalcoholic steatohepatitis", Current Pharmacology Reports, 3, 92-100 (2017).

7- E. Gäbele, K. Dostert, C. Hofmann, R. Wiest, J. Schölmerich, C. Hellerbrand and F. Obermeier, "DSS induced colitis increases portal LPS levels and enhances hepatic inflammation and fibrogenesis in experimental NASH", Journal of Hepatology, 55, 1391-1399 (2011).

8- K. Rahman, C. Desai, S. S. Iyer, N. E. Thorn, P. Kumar, Y. Liu, T. Smith, A. S. Neish, H. Li, S. Tan, P. Wu, X. Liu, Y. Yu, A. B. Farris, A. Nusrat, C. A. Parkos and F. A. Anania, "Loss of junctional adhesion molecule a promotes severe steatohepatitis in mice on a diet high in saturated fat, fructose, and cholesterol", Gastroenterology, 151, 733-746.E12 (2016).

9- R. M. Abdou, L. Zhu, R. D. Baker and S. S. Baker, "Gut microbiota of nonalcoholic fatty liver disease", Digestive Diseases and Sciences, 61, 1268-1281 (2016).

10- T. Goto, M. Itoh, T. Suganami, S. Kanai, I. Shirakawa, T. Sakai, M. Asakawa, T. Yoneyama, T. Kai and Y. Ogawa, "Obeticholic acid protects against hepatocyte death and liver fibrosis in a murine model of nonalcoholic steatohepatitis", Scientific Reports, 8, Article No. 8157 (2018).

11- B. Kong, J. P. Luyendyk, O. Tawfik and G. L. Guo, "Farnesoid $\mathrm{x}$ receptor deficiency induces nonalcoholic steatohepatitis in low-density lipoprotein receptor-knockout mice fed a high-fat diet", Journal of Pharmacology and Experimental Therapeutics, 328, 116-122 (2009).

12- P. M. Rodrigues, M. B. Afonso, A. L. Simaõ, C. C. Carvalho, A. Trindade, A. Duarte, P. M. Borralho, M. V. MacHado, H. Cortez-Pinto, C. M. Rodrigues and R.
E. Castro, "MiR-21 ablation and obeticholic acid ameliorate nonalcoholic steatohepatitis in mice", Cell Death and Disease, 8, e2748 (2017).

13- E. Seki and B. Schnabl, "Role of innate immunity and the microbiota in liver fibrosis: Crosstalk between the liver and gut", The Journal of Physiology, 590, 447-458 (2012).

14- Y. Ilan, "Leaky gut and the liver: A role for bacterial translocation in nonalcoholic steatohepatitis", World Journal of Gastroenterology, 18, 2609-2618 (2012).

15- D. E. Fouts, M. Torralba, K. E. Nelson, D. A. Brenner and B. Schnabl, "Bacterial translocation and changes in the intestinal microbiome in mouse models of liver disease", Journal of Hepatology, 56, 1283-1292 (2012).

16- J. Y. L. Chiang, "Bile acid metabolism and signaling", Comprehensive Physiology, 3, 1191-1212 (2013).

17- Y. fan Nie, J. Hu and X. hua Yan, "Crosstalk between bile acids and intestinal microbiota in host metabolism and health", Journal of Zhejiang University: Science B, 16, 436-446 (2015).

18- A. H. Ali, E. J. Carey and K. D. Lindor, "Recent advances in the development of farnesoid X receptor agonists", Annals of Translational Medicine, 3, (2015).

19- S. Zhang, J. Wang, Q. Liu and D. C. Harnish, "Farnesoid $\mathrm{X}$ receptor agonist WAY-362450 attenuates liver inflammation and fibrosis in murine model of non-alcoholic steatohepatitis", Journal of Hepatology, 51, 380-388 (2009).

20- W. Wu, X. Liu, X. Peng, R. Xue, L. Ji, X. Shen, S. Chen, J. Gu and S. Zhang, "Bile acids override steatosis in farnesoid $\mathrm{X}$ receptor deficient mice in a model of nonalcoholic steatohepatitis", Biochemical and Biophysical Research Communications, 448, 50-55 (2014).

21- S. Peddicord, "FDA approves Ocaliva for rare, chronic liver disease", FDA News Release, (2016).

22- F. G. Schaap, M. Trauner and P. L. M. Jansen, "Bile acid receptors as targets for drug development", Nature Reviews Gastroenterology \& Hepatology, 11, 5567 (2014). 
23- M. Kugelmas, D. B. Hill, B. Vivian, L. Marsano and C. J. McClain, "Cytokines and NASH: A pilot study of the effects of lifestyle modification and vitamin E", Hepatology, 38, 413-419 (2003).

24- N. Matsuzawa, T. Takamura, S. Kurita, H. Misu, T. Ota, H. Ando, M. Yokoyama, M. Honda, Y. Zen, Y. Nakanuma, K. I. Miyamoto and S. Kaneko, "Lipid-induced oxidative stress causes steatohepatitis in mice fed an atherogenic diet", Hepatology, 46, 1392-1403 (2007).

25- A. Karlsson, A. Jägervall, M. Pettersson, A. K. Andersson, P. G. Gillberg and S. Melgar, "Dextran sulphate sodium induces acute colitis and alters hepatic function in hamsters", International Immunopharmacology, 8, 20-27 (2008).

26- M. G. Neuman, L. B. Cohen and R. M. Nanau Bsc, "Biomarkers in nonalcoholic fatty liver disease", Canadian Journal of Gastroenterology and Hepatology, 28 (11), 607-618. (2014).

27- V. Kumar, "NKT-cell subsets: Promoters and protectors in inflammatory liver disease", Journal of Hepatology, 59, 618620 (2013).

28- C. J. Horras, C. L. Lamb and K. A. Mitchell, "Regulation of hepatocyte fate by interferon- $\gamma$ ", Cytokine and Growth Factor Reviews, 22, 35-43 (2011).

29- M. Sewnath, "Endogenous interferon $\gamma$ protects against cholestatic liver injury in mice", Hepatology, 36, 1466-1477 (2002).

30- D. Holmes, "Paradigm shift in the immunopathogenesis of NAFLD", Nature Reviews Endocrinology, 13, 500 (2017).

31- B. Knight, R. Lim, G. C. Yeoh and J. K. Olynyk, "Interferon- $\gamma$ exacerbates liver damage, the hepatic progenitor cell response and fibrosis in a mouse model of chronic liver injury", Journal of Hepatology, 47, 826-833 (2007).

32- X.-Y. Luo, T. Takahara, K. Kawai, M. Fujino, T. Sugiyama, K. Tsuneyama, K. Tsukada, S. Nakae, L. Zhong and X.-K. $\mathrm{Li}$, "IFN- $\gamma$ deficiency attenuates hepatic inflammation and fibrosis in a steatohepatitis model induced by a methionine- and choline-deficient high-fat diet", American Journal of PhysiologyGastrointestinal and Liver Physiology, 305, G891-G899 (2013).

33- Y. Mao, F. Yu, J. Wang, C. Guo and X. Fan, "Autophagy: A new target for nonalcoholic fatty liver disease therapy", Hepatic Medicine: Evidence and Research, 8, 27-37 (2016).

34- A. González-Rodríguez, R. Mayoral, N. Agra, M. P. Valdecantos, V. Pardo, M. E. Miquilena-Colina, J. Vargas-Castrillón, O. lo Iacono, M. Corazzari, G. M. Fimia, M. Piacentini, J. Muntané, L. Boscá, C. García-Monzón, P. Martín-Sanz and A. M. Valverde, "Impaired autophagic flux is associated with increased endoplasmic reticulum stress during the development of NAFLD", Cell Death and Disease, 5, e1179 (2014).

35- M. J. Czaja, "Function of autophagy in nonalcoholic fatty liver disease", Digestive Diseases and Sciences, 61, 1304-1313 (2016).

36- X. Xiong, Y. Ren, Y. Cui, R. Li, C. Wang and Y. Zhang, "Obeticholic acid protects mice against lipopolysaccharide-induced liver injury and inflammation", Biomedicine and Pharmacotherapy, 96, 1292-1298 (2017).

37- J. M. Lee, M. Wagner, R. Xiao, K. H. Kim, D. Feng, M. A. Lazar and D. D. Moore, "Nutrient-sensing nuclear receptors coordinate autophagy", Nature, 516, 112-115 (2014).

38- S. Seok, T. Fu, S. E. Choi, Y. Li, R. Zhu, S. Kumar, X. Sun, G. Yoon, Y. Kang, W. Zhong, J. Ma, B. Kemper and J. K. Kemper, "Transcriptional regulation of autophagy by an FXR-CREB axis", Nature, 516, 108-111 (2014). 


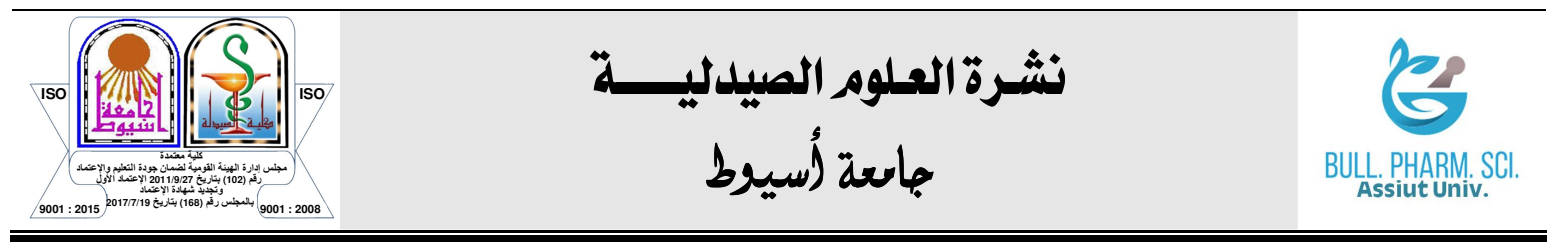

\section{دراسة تأثير تحفيز مستقبل FXR على الالتهام الأتي بالكبا في نموذج تجريبي لمرض التهاب الكبد الادهني}

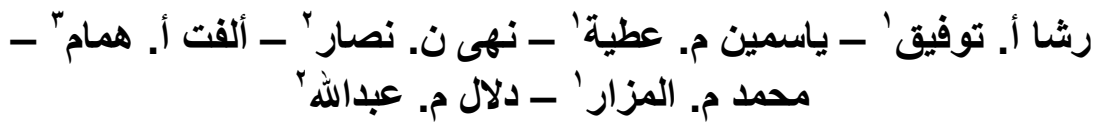

' قسم الفارماكولوجى ، كلية الصيدلة ، الجامعة البريطانية في مصر ، القاهرة ، مصر

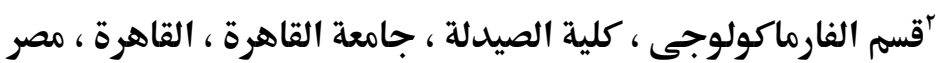

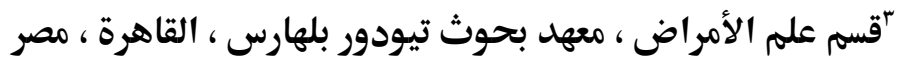

تثير العديد من الأدلة إلى قدرة اضطر اب أنواع بكتيريا المعدة ، فرط نمو بكتيريا المعدة والتغير

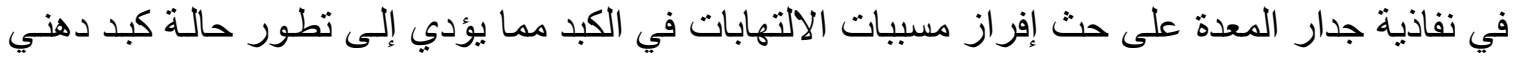

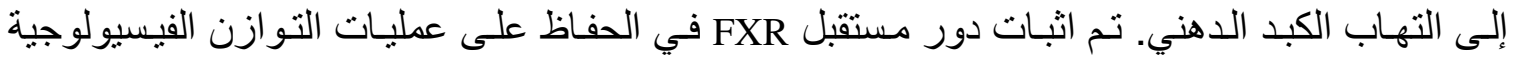

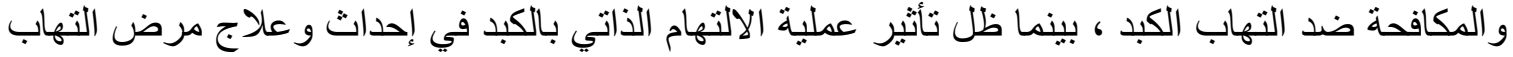

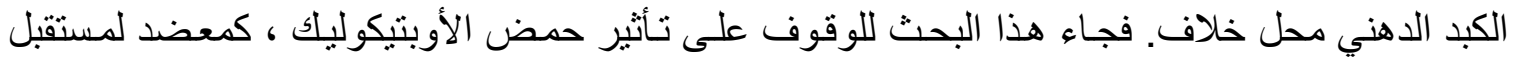

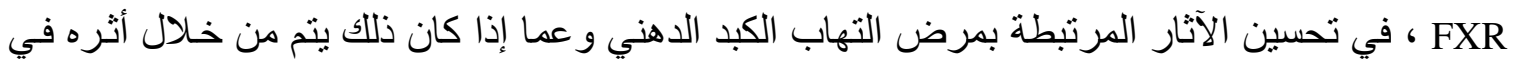
تحفيز عملية الالتهام الذاتي بالكبد.

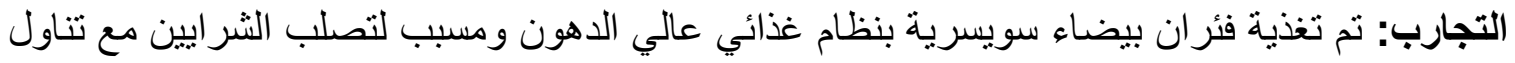

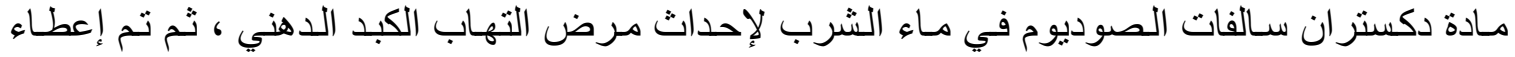

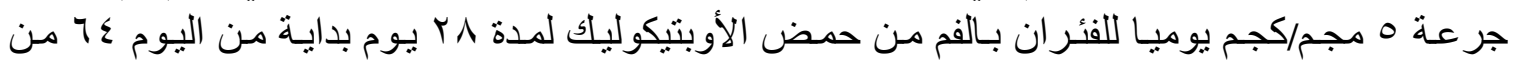

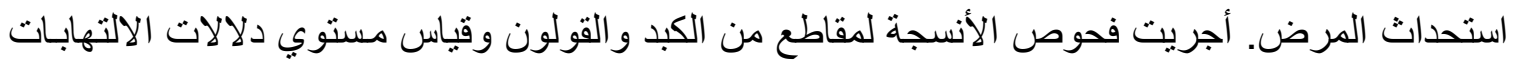

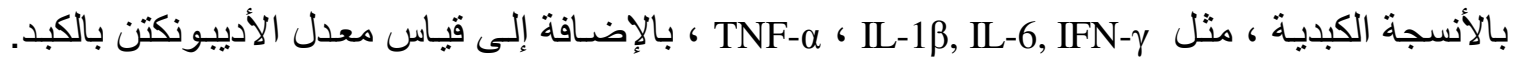

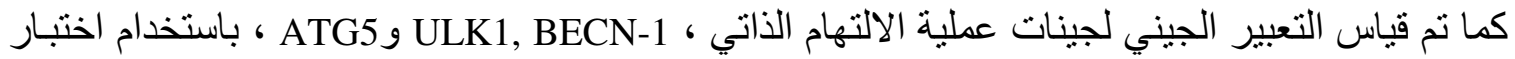
RT-PCR

النتائج: بعد إعطاء حمض الأوبتيكوليك ، تم علاج تغير ات الأنسجة في مقاطع الكبد و القولون لتصبح

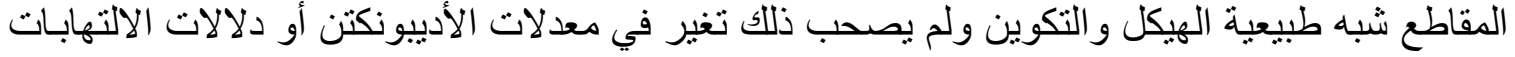

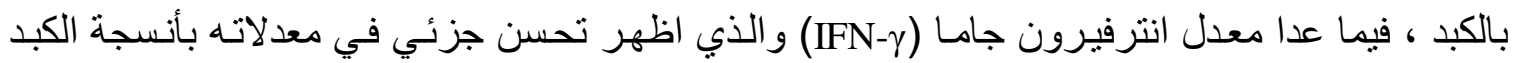

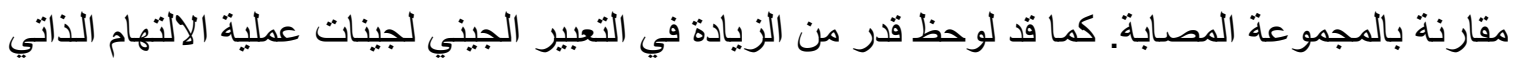
، ULK1 and BECN-1 ، الاستتناج: لحمض الأوبتيكوليك قدرة على علاج مرض التهاب الكبد الدهني من خلال تأثيره الجزئي في حث الالتهام الذاتي وتعزيز إفراز الانترفيرون جاما في خلايا الإنا الكبد. 